University of Nebraska - Lincoln

DigitalCommons@University of Nebraska - Lincoln

Faculty Publications from Nebraska Center for

Materials and Nanoscience, Nebraska Center Materials and Nanoscience

for (NCMN)

3-28-2007

\title{
Calculations of spin-disorder resistivity from first principles
}

\author{
Aleksander L. Wysocki \\ University of Nebraska-Lincoln, awysocki@iastate.edu \\ Kirill D. Belashchenko \\ University of Nebraska-Lincoln, belashchenko@unl.edu \\ Julian P. Velev \\ University of Nebraska-Lincoln, julian.velev@unl.edu \\ Mark van Schilfgaarde \\ Arizona State University, mark.van_schilfgaarde@kcl.ac.uk
}

Follow this and additional works at: https://digitalcommons.unl.edu/cmrafacpub

Part of the Nanoscience and Nanotechnology Commons

Wysocki, Aleksander L.; Belashchenko, Kirill D.; Velev, Julian P.; and van Schilfgaarde, Mark, "Calculations of spin-disorder resistivity from first principles" (2007). Faculty Publications from Nebraska Center for Materials and Nanoscience. 19.

https://digitalcommons.unl.edu/cmrafacpub/19

This Article is brought to you for free and open access by the Materials and Nanoscience, Nebraska Center for (NCMN) at DigitalCommons@University of Nebraska - Lincoln. It has been accepted for inclusion in Faculty Publications from Nebraska Center for Materials and Nanoscience by an authorized administrator of DigitalCommons@University of Nebraska - Lincoln. 


\title{
Calculations of spin-disorder resistivity from first principles
}

\author{
A. L. Wysocki, ${ }^{\text {a) }}$ K. D. Belashchenko, and J. P. Velev \\ Department of Physics and Astronomy, University of Nebraska-Lincoln, Lincoln, Nebraska 68588 and \\ Nebraska Center for Materials and Nanoscience, University of Nebraska-Lincoln, Lincoln, Nebraska 68588
}

\author{
M. van Schilfgaarde \\ Department of Chemical and Materials Engineering, Arizona State University, Tempe, Arizona 85287
}

(Presented on 10 January 2007; received 31 October 2006; accepted 8 November 2006; published online 28 March 2007)

\begin{abstract}
Spin-disorder resistivity of Fe and Ni is studied using the noncollinear density functional theory. The Landauer conductance is averaged over random disorder configurations and fitted to Ohm's law. The distribution function is approximated by the mean-field theory. The dependence of spin-disorder resistivity on magnetization in $\mathrm{Fe}$ is found to be in excellent agreement with the results for the isotropic $s$ - $d$ model. In the fully disordered state, spin-disorder resistivity for $\mathrm{Fe}$ is close to experiment, while for fcc $\mathrm{Ni}$ it exceeds the experimental value by a factor of 2.3 . This result indicates strong magnetic short-range order in $\mathrm{Ni}$ at the Curie temperature. (C) 2007 American Institute of Physics. [DOI: 10.1063/1.2670472]
\end{abstract}

The effects of thermal spin disorder on the transport properties of ferromagnets are of interest for both fundamental and practical reasons. First, studies of spin-disorder resistivity may provide important information on magnetic shortrange order in ferromagnets, which is an intrinsic thermodynamic feature of spin disorder. Second, spin disorder intermixes the spin channels and thereby introduces finite spin diffusion length in ferromagnets. Together with scattering on interface spin disorder and spin-orbit scattering, this effect degrades the magnetoresistance of magnetic tunnel junctions and spin valves, which are used in magnetic field sensors and magnetic random-access memories.

A large amount of experimental data is available on the electric resistivity of ferromagnetic metals, most of which was collected many years ago. ${ }^{1}$ In these metals the resistivity has an "anomalous" contribution which contains signatures of magnetic phase transitions. This magnetic contribution $\rho_{\text {mag }}$ may be extracted from the measured resistivity assuming that Matthiessen's rule is valid, and that the anomalous contribution is temperature independent well above the Curie temperature. $^{2}$

The anomalous resistivity $\rho_{\text {mag }}$ may be attributed to spindisorder scattering, which was studied using the $s-d$ model Hamiltonian by many authors. ${ }^{3-6}$ This approach assumes that $3 d$ electrons in transition metals are localized at atomic sites, while the conductivity is due to mobile $4 s$ electrons forming an itinerant band and coupled to the $d$ electrons by exchange interaction. At finite temperatures the directions of the $d$-electron spins fluctuate, and the conducting $s$ electrons scatter from the inhomogeneous exchange potential.

As the temperature is increased toward the Curie temperature $T_{\mathrm{C}}$, the spins become more disordered, and $\rho_{\mathrm{mag}}$ quickly increases, sometimes surpassing the phonon contribution. At elevated temperatures the $s$ - $d$ model calculations of $\rho_{\text {mag }}$ were done using the mean-field approximation for

${ }^{a)}$ Electronic mail: awysocki@bigred.unl.edu magnetic thermodynamics. In this approximation, the spins are completely disordered above $T_{\mathrm{C}}$, and hence $\rho_{\text {mag }}$ is constant. In the Born approximation below $T_{\mathrm{C}}$ it declines as

$$
\rho_{\mathrm{mag}}(T)=\rho_{\mathrm{mag}}\left(T_{\mathrm{C}}\right)\left[1-M^{2}(T) / M^{2}(0)\right]
$$

where $M(T)$ is the magnetization at temperature $T^{3,4,6}$ In general, $\rho_{\text {mag }}$ is sensitive to magnetic short-range rather than long-range order. ${ }^{7}$ Quantitative comparison of the calculations of $\rho_{\text {mag }}(T)$ with experiment can thus provide valuable information on magnetic short-range order, which may be very strong in itinerant ferromagnets such as $\mathrm{Ni}^{8}$

Unfortunately, since the $s-d$ model assumes an isotropic conduction band, it cannot predict the magnitude of $\rho_{\text {mag }}\left(T_{\mathrm{C}}\right)$ of specific materials. Moreover, treating the $3 d$ electrons as localized appears to be an inadequate approximation for computing the spin-disorder resistivity, because the dominant contribution comes from interband $(s-d)$ rather than intraband $(s-s)$ scattering transitions. This conclusion was made by Goodings ${ }^{9}$ who solved the Boltzmann equation for the isotropic two-band model. Although this model is not applicable to transition metals with complex Fermi surfaces, it clearly highlights the sensitivity of the spin-disorder resistivity to the band structure. A parameter-free approach based on the density functional theory is clearly needed for quantitative comparison with experiment.

In this paper we calculate $\rho_{\text {mag }}$ for iron and nickel from first principles using the Kubo-Landauer formalism implemented in the tight-binding linear muffin-tin orbital (TBLMTO) method within the atomic sphere approximation, which was extended to noncollinear systems. Our treatment of spin disorder is based on the adiabatic approximation, which assumes that the low-lying excitations of the magnet are well represented by the states where the magnetic moments at different atomic sites are rigidly rotated off of the magnetization axis. ${ }^{10}$ The state of the system is assumed to be the ground state constrained by these rotations. For a given noncollinear spin configuration, one can thus construct 


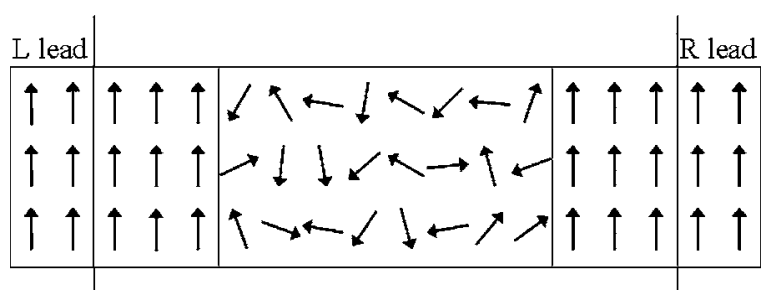

FIG. 1. The schematic picture of the system used in the calculations. Vertical lines indicate the embedding planes.

a TB-LMTO Hamiltonian or Green's function matrix (which are no more diagonal in spin space). ${ }^{11}$ We used the local spin density approximation and frozen atomic potentials taken from the ferromagnetic ground state. The basis set included the states up to $l_{\max }=2$ for both $\mathrm{Fe}$ and $\mathrm{Ni}(s, p$, and $d$ orbitals).

The conductance was calculated using principal-layer Green's function technique and the Kubo-Landauer formalism. ${ }^{12,13}$ We consider a ferromagnetic layer FM(D) with spin disorder placed between two magnetically ordered leads $\mathrm{FM}(\mathrm{O})$ made of the same material, as seen in Fig. 1. As usual in the principal-layer technique, the "active region" consists of the $\mathrm{FM}(\mathrm{D})$ layer plus a few monolayers of $\mathrm{FM}(\mathrm{O})$ on each side.

Because we use frozen atomic potentials to make calculations practical, care needs to be taken to ensure local charge neutrality. Indeed, one can view $\mathrm{FM}(\mathrm{D})$ and $\mathrm{FM}(\mathrm{O})$ as different materials which have different Fermi levels that must normally be matched by the contact potential. Frozen atomic potentials do not allow this to happen, and therefore we introduced a compensating constant potential shift in the disordered region which mimics the contact potential. This shift was found so that the charge in the central part of FM(D) averaged over disorder realizations is zero. Note that no matter how the $\mathrm{FM}(\mathrm{O}) / \mathrm{FM}(\mathrm{D})$ interfaces are treated (selfconsistently or not), they add contact resistances to the circuit. However, since the resistivity of the FM(D) material is extracted from the dependence of the resistance on the FM(D) thickness in the Ohmic limit, it does not depend on the interfaces, and therefore our crude treatment of the interfaces does not introduce any error.

First we considered the paramagnetic state with no magnetic short-range order (completely uncorrelated spins with uniform angular distribution function). For the given thickness of the FM(D) layer, the resistance of the system was averaged over several disorder configurations (typically 12). As seen in Fig. 2, almost perfect Ohmic behavior was found for Fe down to the smallest thicknesses for the $4 \times 4$ lateral cell (16 atoms per layer). The convergence with respect to the lateral cell size was also checked. We found that for paramagnetic $\mathrm{Fe} \rho_{\mathrm{mag}}$ is quite insensitive to this size, as seen in Table I. For Ni the thickness dependence of $\rho_{\mathrm{mag}}$ is also fairly close to linear for the $2 \times 2$ lateral cell (eight atoms per layer).

The calculated $\rho_{\text {mag }}$ for Fe (see Table I) is about $20 \%$ greater than the experimental estimate. ${ }^{2}$ This result indicates that magnetic short-range order in $\mathrm{Fe}$ at $T_{\mathrm{C}}$ is weak. However, for the similar model of fcc Ni $\rho_{\text {mag }}$ is nearly 2.3 times

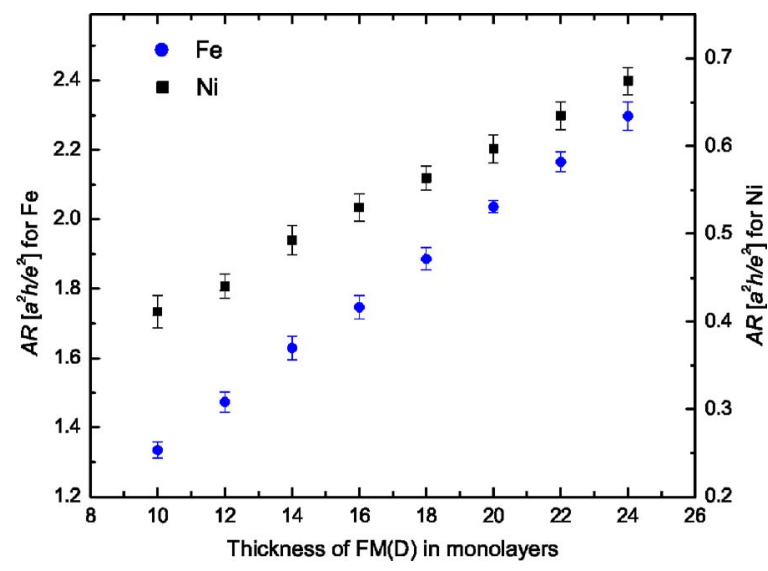

FIG. 2. The area-resistance product $A R$ of the $F M(O) / F M(D) / F M(O)$ systems as a function of the thickness of the FM(D) layer for bcc Fe and fcc Ni in the paramagnetic state. The lateral cell sizes were $4 \times 4$ for $\mathrm{Fe}$ and 2 $\times 2$ for $\mathrm{Ni}$, with edges along the [100] directions. The error bars denote standard deviations of AR due to the finite number of spin-disorder configurations.

greater than the experimental estimate. This large difference indicates either very strong magnetic short-range order, or a strongly reduced local moment at $T \sim T_{\mathrm{C}}$.

Further, we calculated $\rho_{\text {mag }}$ for ferromagnetic Fe in a range of temperatures. The spin configurations in the disordered $\mathrm{Fe}$ layer were generated using the mean-field theory, i.e., with an uncorrelated distribution function given by

$$
p(\theta) \propto e^{-\mathbf{H}_{\text {eff }} \boldsymbol{\mu} / T}, \quad H_{\text {eff }}(T)=\frac{3 M(T) T_{\mathrm{C}}}{\mu M(0)},
$$

where $\theta$ is the angle between the spin and the magnetization axis, $\mu$ is the magnetic moment at each site, $M(T)$ is the magnetization at temperature $T$ in the mean-field approximation, and $\mathbf{H}_{\text {eff }}$ is the effective Weiss field.

We used $4 \times 4$ lateral cells for all temperatures and thicknesses. The Ohmic limit (linear dependence of the conductance on length) was achieved for all temperatures down to about $T_{\mathrm{C}} / 3$. This behavior agrees with what one would expect from simple mean free path considerations. If we estimate the mean free path as $l=\frac{3}{4} \mathrm{AR}_{\mathrm{bal}} / \rho$, where $\mathrm{AR}_{\mathrm{bal}}$ is the ballistic area-resistance product (this formula is exact for the free-electron model), we find that $l$ does not exceed the lateral cell size in this temperature range. Therefore, quantum fluctuations of the conductance are small, and the transport is diffusive. Another indication of the Ohmic behavior comes from the distribution of the current over the spin channels. The conductance is a sum of four partial conductances, $G_{\uparrow \uparrow}, G_{\downarrow \downarrow}, G_{\uparrow \downarrow}, G_{\downarrow \uparrow}$. Spin-conserving and spin-flip scattering have similar rates in our spin-disorder problem, and therefore

TABLE I. Spin-disorder resistivity $\rho_{\text {mag }}$ in $\mu \Omega \mathrm{cm}$ for paramagnetic bcc Fe and fcc Ni. The calculated values are given for different lateral cell sizes with edges along the [100] directions. The experimental values are the hightemperature asymptotes taken from Ref. 2.

\begin{tabular}{ccccc}
\hline \hline Lateral cell size & $2 \times 2$ & $3 \times 3$ & $4 \times 4$ & Expt. \\
\hline $\mathrm{Fe}$ & 105.5 & 100.1 & 101.9 & 80 \\
$\mathrm{Ni}$ & 34 & & & 15 \\
\hline
\end{tabular}




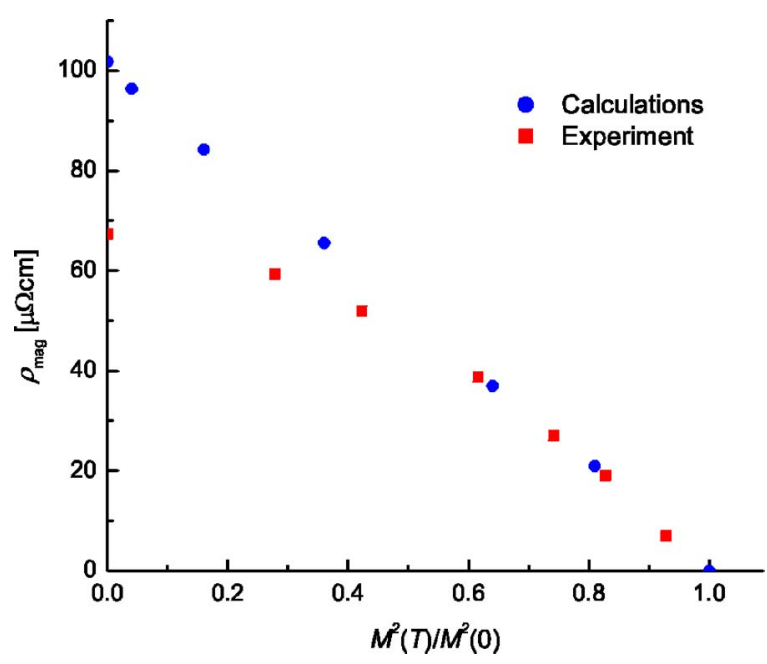

FIG. 3. Spin-disorder resistivity of Fe plotted against $M^{2}(T)$. The experimental data for $\rho_{\text {mag }}(T)$ were taken from Ref. 2, and those for $M(T)$ from Ref. 14.

the electrons lose memory of their spin over their mean free path. Therefore, in the Ohmic limit the partial conductances must be proportional to the number of channels in the left and right leads for the corresponding spin channels: $G_{\sigma \sigma^{\prime}}$ $\propto M_{\sigma}^{L} M_{\sigma^{\prime}}^{R}$. This behavior was indeed observed down to $T$ $\sim T_{\mathrm{C}} / 3$.

The results of $\rho_{\text {mag }}(T)$ calculations for Fe are plotted in Fig. 3 versus $M^{2}(T)$ along with the experimental data taken from Ref. 2. Surprisingly, the calculated results, which are based on first-principles electronic structure and do not involve perturbation theory, show that the temperature dependence agrees with Eq. (1) with a rather high accuracy. This striking agreement suggests that the temperature dependence of $\rho_{\mathrm{mag}}$ is quite insensitive to the shape of the Fermi surface.

In the low-temperature region (large $M$ ) the magnetic excitations are in reality dominated by spin waves, and the mean-field theory is inapplicable. Therefore, the fact that the theoretical and experimental curves in Fig. 3 have the same slope in this region is probably accidental (the extraction of $\rho_{\text {mag }}$ using Matthiessen's rule is also unreliable here). In the high-temperature region the comparison is more meaningful, and here we see that the calculated results are quite close to experiment at $M^{2}(T) / M^{2}(0) \sim 0.5$ and somewhat diverge as the temperature approaches $T_{\mathrm{C}}$. There may be several reasons for this behavior, including the dependence of the local moment on temperature which we neglected, and also the presence of magnetic short-range order. The resistivity is sensitive to short-range rather than long-range order, ${ }^{7}$ and the short-range order depends less strongly on the temperature. Therefore, the deviation of the theoretical curve from experiment close to $T_{\mathrm{C}}$ is expected. Quantitative analysis of these effects deserves further investigation.

In conclusion, we calculated the spin-disorder contribution to resistivity for iron and nickel from first principles. Comparison with experimental data suggests that magnetic short-range order is weak in Fe and strong in Ni. The analysis of spin-disorder resistivity can thus provide important information on magnetic short-range order in magnetic metals. We also studied the temperature dependence of spindisorder resistivity in Fe assuming mean-field thermodynamics. The resistivity depends linearly on $M^{2}(T) / M^{2}(0)$ in agreement with the predictions of the isotropic $s-d$ model.

We are grateful to V. P. Antropov and E. Y. Tsymbal for useful discussions. This work was supported by the Nebraska Research Initiative and NSF MRSEC. It was completed utilizing the Research Computing Facility of the University of Nebraska-Lincoln.

${ }^{1}$ B. R. Coles, Adv. Phys. 7, 40 (1958).

${ }^{2}$ R. J. Weiss and A. S. Marotta, J. Phys. Chem. Solids 9, 302 (1959).

${ }^{3}$ P. G. de Gennes and J. Friedel, J. Phys. Chem. Solids 4, 71 (1958).

${ }^{4}$ I. Mannari, Prog. Theor. Phys. 26, 51 (1961).

${ }^{5}$ S. V. Vonsovskii, Magnetism (Halsted, New York, 1974).

${ }^{6}$ T. Kasuya, Prog. Theor. Phys. 16, 58 (1956).

${ }^{7}$ M. E. Fisher and J. S. Langer, Phys. Rev. Lett. 20, 667 (1968).

${ }^{8}$ V. P. Antropov, Phys. Rev. B 72, 140406 (2005).

${ }^{9}$ D. A. Goodings, Phys. Rev. 132, 542 (1963).

${ }^{10}$ B. L. Gyorffy, A. J. Pindor, J. Staunton, G. M. Stocks, and H. Winter, J. Phys. F: Met. Phys. 15, 1337 (1985).

${ }^{11}$ V. P. Antropov, M. I. Katsnelson, B. N. Harmon, M. van Schilfgaarde, and D. Kuznezov, Phys. Rev. B 54, 1019 (1996).

${ }^{12}$ I. Turek, V. Drchal, J. Kudrnovský, M. Šob, and P. Weinberger, Electronic Structure of Disordered Alloys, Surfaces and Interfaces (Kluwer, Boston, 1997).

${ }^{13}$ J. Kudrnovský, V. Drchal, C. Blaas, P. Weinberger, I. Turek, and P. Bruno, Phys. Rev. B 62, 15084 (2000).

${ }^{14}$ J. Crangle and G. M. Goodman, Proc. R. Soc. London, Ser. A 321, 477 (1971). 